Published in final edited form as:

Surg Oncol Clin N Am. 2014 July ; 23(3): 505-523. doi:10.1016/j.soc.2014.03.006.

\title{
Neoadjuvant therapy in the treatment of breast cancer
}

\author{
Mediget Teshome, M.D. and Kelly K. Hunt, M.D. \\ Department of Surgical Oncology, MD Anderson Cancer Center, Houston, TX
}

\section{Synopsis}

Neoadjuvant systemic therapy in the treatment of breast cancer was initially employed for patients with inoperable disease. Over the past several decades this treatment approach has proved beneficial in many other patients including those with early-stage, operable breast cancer. Several randomized prospective studies have shown comparable survival rates when compared with adjuvant systemic therapy. Additionally, neoadjuvant chemotherapy can decrease the tumor burden facilitating breast conservation in selected patients without significant increases in local recurrence. Response to therapy has proven to be a strong predictor of outcome, with patients achieving pathologic complete response ( $\mathrm{pCR}$ ) demonstrating improved survival compared with those achieving less than a pCR. Furthermore, molecular subtype analysis has shown improved response following neoadjuvant chemotherapy in certain tumor types providing patients with the most aggressive subtypes a chance at cure with targeted therapies. In particular, targeting the HER2-positive subtype with trastuzumab and other HER2-directed therapies has markedly improved the outcome in these patients. Conversely, the early recognition of poor responders is important in limiting the toxicity of ineffective therapy and altering management. Neoadjuvant endocrine therapy in postmenopausal women with hormone receptor-positive tumors consistently decreases tumor size improving rates of breast conservation. Aromatase inhibitors have demonstrated superiority to tamoxifen with improved response and favorable toxicity profiles. Imaging modalities have shown promise in predicting patients with $\mathrm{pCR}$, however they have not yet eliminated the need for surgical intervention. Less invasive surgical strategies such as breast conserving surgery and sentinel lymph node dissection have been shown to be safe following neoadjuvant chemotherapy in selected patients. A multidisciplinary approach with primary systemic therapy when indicated, improves the likelihood for breast conservation, provides a window into tumor biology and predicts patient outcomes.

\section{Keywords}

breast cancer; neoadjuvant chemotherapy; preoperative chemotherapy

(C) 2014 Elsevier Inc. All rights reserved.

Address correspondence to: Dr. Hunt at The University of Texas MD Anderson Cancer Center, 1400 Pressler Street, Unit 1484, Houston, Texas 77030. Phone: 713-792-7216. Facsimile: 713-792-0722. khunt@ mdanderson.org.

The authors have nothing to disclose

Publisher's Disclaimer: This is a PDF file of an unedited manuscript that has been accepted for publication. As a service to our customers we are providing this early version of the manuscript. The manuscript will undergo copyediting, typesetting, and review of the resulting proof before it is published in its final citable form. Please note that during the production process errors may be discovered which could affect the content, and all legal disclaimers that apply to the journal pertain. 


\section{Introduction}

The treatment of breast cancer has evolved from a primary surgical approach with focus on local-regional control to multidisciplinary management with emphasis on systemic therapy resulting in significantly improved survival. The optimal timing of systemic therapy relative to operative management has been studied for several decades with strong evidence supporting a neoadjuvant approach to systemic therapy in certain populations. Neoadjuvant therapy implies initiation of systemic therapy prior to definitive local-regional management. It is also referred to as primary systemic therapy or preoperative chemotherapy. While historically reserved for patients with inoperable disease, contemporary management of breast cancer involves a neoadjuvant approach for patients with inflammatory breast cancer, locally advanced disease and selected patients with early-stage, operable breast cancer.

Neoadjuvant chemotherapy has emerged as a powerful treatment modality with individualized prognostic significance based on response to therapy.

\section{Rationale for neoadjuvant chemotherapy}

The paradigm shift from adjuvant to neoadjuvant chemotherapy is rooted in observations of tumor kinetics and the hypothesis of micrometastatic disease present in the early stages of breast malignancy.[1] Fisher performed animal studies and observed a change in tumor kinetics with increased cellular proliferation at metastatic sites after resection of the primary tumor.[2] Another concern was resistance to chemotherapy with increased tumor growth resulting in increased drug-resistant variants suggesting the optimal time for chemotherapy administration was early after diagnosis.[3] These observations along with multiple clinical studies showing benefit in decreasing the primary tumor burden prompted randomized trials examining the potential survival benefit of neoadjuvant chemotherapy in treating disseminated micrometastatic disease to improve survival. Although this survival advantage was not ultimately demonstrated, decrease in the primary tumor burden converted some patients to operative candidates and facilitated breast conservation in at least $25 \%$. In addition, insights into prognosis based on tumor response arose as significant clinical implications of neoadjuvant chemotherapy.

\section{Comparison of neoadjuvant to adjuvant systemic therapy}

Initiated in 1988, the National Surgical Adjuvant Breast and Bowel Project (NSABP) B-18 protocol was one of the earliest and largest randomized trials of neoadjuvant vs. adjuvant chemotherapy for women with operable breast cancer. This study randomized 1,523 women to surgery followed by four cycles of AC (doxorubicin $60 \mathrm{mg} / \mathrm{m}^{2}$ and cyclophosphamide $600 \mathrm{mg} / \mathrm{m}^{2}$ ) or preoperative AC followed by surgery. The planned surgical approach was determined prior to randomization. All women who were over 50 years of age were treated with tamoxifen after the completion of chemotherapy and those treated with breast conservation received whole breast radiation therapy. The primary objective of the B-18 study was to determine if neoadjuvant chemotherapy resulted in improved overall survival (OS) and disease-free survival (DFS) as compared to delivery of the same chemotherapy in the adjuvant setting. Secondary aims were to evaluate the effect of neoadjuvant chemotherapy on response in the primary tumor, response in axillary lymph nodes and 
facilitation of breast conservation.[4] The investigators found no difference in overall survival or disease-free survival in women treated with preoperative chemotherapy as compared to those treated with adjuvant chemotherapy. This finding has persisted after 16 years of followup.[5] Patients in the preoperative AC group showed 79\% objective clinical response, $43 \%$ clinical partial response, $36 \%$ clinical complete response and $13 \%$ pathologic complete response after treatment. This group also had increased incidence of pathologically negative axillary nodal disease and rates of breast conservation. There was a non-statistically significant trend in increased ipsilateral breast tumor recurrence following preoperative chemotherapy. Pathologic complete response and post-treatment pathological negative nodal status emerged as strong predictors of both overall survival and disease-free survival.

Similar to the NSABP B-18 trial, the European Organization Research and Treatment of Cancer (EORTC) Trial 10902 examined neoadjuvant chemotherapy in women with operable breast cancer as compared to adjuvant therapy.[6] This study randomized 698 women to 4 cycles of preoperative FEC (fluorouracil $600 \mathrm{mg} / \mathrm{m}^{2}$, epirubicin $60 \mathrm{mg} / \mathrm{m}^{2}$ and cyclophosphamide $600 \mathrm{mg} / \mathrm{m}^{2}$ ) followed by surgery or the same regimen postoperatively. The investigators found no difference between the treatment groups in overall survival, progression-free survival or time to loco-regional recurrence. They observed an overall response rate of $49 \%$ in the preoperative chemotherapy group with $6.6 \%$ of patients experiencing complete clinical response and $3.7 \%$ with pathologic complete response. They also showed an increased survival advantage with pathologic complete response that was not demonstrated with complete clinical response. There was a significant down-sizing in clinical tumor size to $<2 \mathrm{~cm}$ in the preoperative arm from $14 \%$ at diagnosis and $47 \%$ after treatment compared with $14 \%$ and $26 \%$ in the postoperative arm, respectively. In the preoperative group $23 \%$ of patients initially planned for mastectomy underwent breast conservation.

A meta-analysis evaluating nine randomized trials addressing outcomes after neoadjuvant and adjuvant chemotherapy in 3,946 patients reached similar conclusions. Investigators reported no difference in overall survival, disease progression and distant disease recurrence between the two groups. There was a $22 \%$ increased relative risk of loco-regional recurrence with neoadjuvant chemotherapy. While this was a statistically significant finding, it was attributed to three trials in which patients were not obligated to surgery after complete clinical response and received radiation therapy only. This underscores the importance of operative intervention in patients with complete clinical response for maintenance of local control. Pathologic complete response varied from $4 \%$ to $29 \%$ reported in five studies. Additionally, there was a higher rate of breast conservation in the preoperative chemotherapy group.[7] A more recent meta-analysis reviewing fourteen trials randomizing a total of 5,500 women to neoadjuvant chemotherapy or adjuvant chemotherapy confirmed these findings. Loco-regional recurrence, after exclusion of the studies omitting surgical management, was similar among the neoadjuvant and adjuvant chemotherapy groups.[8]

Although these trials did not find increased survival rates following neoadjuvant chemotherapy as hypothesized, they asserted an equivalence of survival benefit with neoadjuvant treatment as compared to the adjuvant setting. Additionally, they established the role for neoadjuvant chemotherapy in decreasing the primary tumor burden and 
facilitating breast conservation without increased risk of local recurrence. Importantly, they demonstrated tumor response as a strong predictor of outcome as evidenced by the association of pathologic complete response and survival.

\section{Introduction of taxanes}

The addition of taxanes to an anthracycline-based regimen in the preoperative setting was evaluated in the NSABP B-27 protocol. This study initiated in 1995, randomized 2,411 women with operable breast cancer to receive four cycles of preoperative AC (doxorubicin $60 \mathrm{mg} / \mathrm{m}^{2}$ and cyclophosphamide $600 \mathrm{mg} / \mathrm{m}^{2}$ ) followed by surgery, preoperative AC and docetaxel $\left(100 \mathrm{mg} / \mathrm{m}^{2}\right)$ followed by surgery and preoperative AC followed by surgery and postoperative docetaxel. The primary objective was to determine if the addition of docetaxel could increase overall survival and disease-free survival. Additional objectives included the effect of preoperative docetaxel in loco-regional control, pathologic complete response, pathologic axillary down-staging and breast conserving surgery.

The investigators found significantly increased rates of objective clinical response ( $86 \%$ vs. $91 \%, \mathrm{p}<0.001)$ and pathologic complete response $(13 \%$ vs. $26 \%, \mathrm{p}<0.001)$ with the addition of neoadjuvant docetaxel to AC. Also observed were decreased rates of pathologically positive nodes and no further increase in the rates of breast conservation. Again, no difference in overall survival and disease-free survival were found with the addition of preoperative or postoperative docetaxel. However, patients who experienced a pCR and were found to have pathologically negative nodes after treatment continued to show an associated survival benefit.[5]

The above studies suggest that it is not the timing of chemotherapy but rather the chemosensitivity and responsiveness of the tumor influencing overall survival.

\section{Prognostic significance of tumor response}

Perhaps the most intriguing implication of preoperative chemotherapy is the ability to observe in vivo tumor chemosensitivity and the prognostic impact of tumor response.

\section{Pathologic complete response}

Neoadjuvant chemotherapy trials have revealed the phenomenon of pathologic complete response (pCR) defined as no residual invasive tumor on pathologic assessment after therapy. Virtually every study examining the impact of pCR after neoadjuvant chemotherapy for breast cancer has demonstrated an associated survival benefit. Furthermore, neoadjuvant chemotherapy has the potential to significantly decrease the axillary nodal disease burden with $23 \%$ of patients converting from clinically node positive to pathologically node negative after treatment with anthracycline-based chemotherapy. Patients who achieve pCR in the primary tumor are more likely to have negative pathologic axillary nodal status and the degree of axillary nodal involvement after chemotherapy is highly predictive of outcome.[9]

Discrepancies exist in the literature in defining pCR with some studies reporting pCR in the breast only and others defining pCR as complete response in the breast and axillary nodes; 
the latter being the currently accepted definition. Importantly, it is only the residual invasive component and not the presence of carcinoma in situ which influences pCR.[10] Factors found to be associated with an increased likelihood of pCR include age $<40$, smaller tumors $(<2.0 \mathrm{~cm})$, ductal histology, high nuclear grade tumors, high rate of cellular proliferation (Ki-67), estrogen receptor negativity, triple negative subtype and HER2-positive disease. [11] Although associated with improved survival overall, a small percentage of patients who achieve pCR will develop disease recurrence and distant disease.[12] Significant factors associated with distant metastasis after pCR include clinical stage IIIB or higher, premenopausal status and $\leq 10$ lymph nodes examined.[13]

The presence of pCR has emerged as a powerful predictor of patient outcome and is utilized as a surrogate endpoint for prognosis in many clinical trials. As such, pCR has entered into contemporary policy with the recent adoption for use in accelerating drug approval by the Food and Drug Administration.[14]

\section{Prognosis after neoadjuvant chemotherapy}

In patients who do not achieve pCR, the residual cancer burden (RCB) can be a useful tool to predict survival. This continuous value incorporates four parameters which hold prognostic significance after neoadjuvant chemotherapy: the primary tumor dimension, cellularity of the invasive cancer, size of largest nodal metastasis and number of positive lymph nodes. Increasing RCB values after chemotherapy are associated with increased risk of 5-year distant relapse. When stratified by extent of residual disease, one study showed rates of distant relapse at 5 years were $2.4 \%$ in those with minimal residual disease (RCB-I) and $53.6 \%$ in those with extensive residual disease (RCB-III). Furthermore, in patients with minimal or no detectable residual disease (RCB-0 or RCB-I) at 5 years the prognosis was similar to those with pCR. Conversely, patients with extensive residual disease (RCB-III) carried a poor prognosis independent of the type of chemotherapy, adjuvant hormonal therapy or pathologic stage. In comparison with post-therapy American Joint Committee on Cancer (AJCC) stage group, the RCB was able to further classify patients with stage II disease into three distinct groups and stage III disease into two distinct groups with different prognoses.[15]

Traditionally, prognosis has been informed by pretreatment tumor-node-metastasis (TNM) clinical stage based on the AJCC system. In the neoadjuvant setting, prognosis is influenced by response to therapy and biologic markers that are not incorporated in traditional staging systems. To address this disparity, a system specifically incorporating the pre-treatment clinical stage and post-treatment pathologic stage after neoadjuvant chemotherapy has been described. The Clinical-Pathologic Scoring (CPS) system stratifies patients into groups with increasing CPS scores associated with decreasing 5-year distant metastasis-free survival and disease-specific survival (DSS). A second system CPS+EG, incorporating ER negative status and nuclear grade 3 tumors as independent risk factors for poor prognosis, was developed.[16] The CPS+EG system provided refinement of 5-year DSS from 23\% to $99 \%$ compared with $61 \%$ to $92 \%$ by clinical stage and $58 \%$ to $95 \%$ by pathologic stage and recurrence-free survival (RFS) with 5-year RFS rates from $15 \%$ to $95 \%$ ( $p<0.001$ ). This

Surg Oncol Clin N Am. Author manuscript; available in PMC 2015 July 01. 
system was subsequently validated in an independent cohort suggesting generalizability among varying institutions with differing populations and practice patterns.[17]

The RCB index and CPS+EG staging system provide further refinement of prognosis by response beyond pCR status to stratify and predict patient outcomes after neoadjuvant chemotherapy. They are prospective tools for clinicians available online that can assist with decision-making and advising patients regarding individualized outcome.

\section{Impact of molecular subtype on pathologic complete response}

Determining molecular tumor subtype (luminal, basal, HER2-positive and normal-like)[18] and approximation of subtype by immunochemistry reveal consistently distinct behavior by subtype in response to neoadjuvant chemotherapy. Generally, more aggressive tumor biology translates into improved pCR with excellent outcome in these patients comparable to more favorable subtypes. A meta-analysis of thirty studies examining pCR after neoadjuvant chemotherapy in 11,695 patients found an overall pooled estimate pCR of $19.2 \%$ when studies with unknown subtype were excluded. Tumor subtype was strongly associated with pCR\% and odds of achieving pCR was seven times higher for those with HER2-positive and five times higher for triple negative subtypes in comparison to the hormone receptor (HR)-positive subtype. Estimates of pCR were $8.3 \%$ in the HR-positive/ HER2-negative subtype, $18.7 \%$ in the HER2-positive/HR-positive subtype, $38.9 \%$ in the HER2-positive/HR-negative subtype and $31.1 \%$ in the triple negative subtype $(\mathrm{p}=0.002)$. [19]

Negative hormone receptor status has been associated with increased chemosensitivity and improved pCR as compared to hormone receptor-positive disease. However, regardless of hormone receptor status, $\mathrm{pCR}$ has been shown to result in improved progression-free survival and overall survival when compared to those who do not achieve pCR.[20] This finding is challenged by a pooled analysis of 6,377 patients treated with anthracyclinetaxane based neoadjuvant chemotherapy demonstrating variation in the prognostic impact of pCR by tumor factors. In low proliferative subgroups, including lobular histology, grade 1 disease and positive hormone receptor status, achieving pCR conferred no predictive power in DFS or OS. In contrast, in those with ductal histology, grade 2 or 3 tumors and negative hormone receptor status, pCR was associated with improved DFS and OS. This suggests that pCR may not be a suitable endpoint in luminal A and luminal B/HER2-negative subtypes. [21]

Some studies have documented a change in hormone receptor or HER2 receptor status when biopsy samples are compared with tissue samples obtained from the same patient after neoadjuvant chemotherapy. Change in hormone receptor status from positive to negative after neoadjuvant chemotherapy was evaluated in 259 patients from Shanghai Cancer Center. This study found a positive to negative change in hormone receptor status in $15.2 \%$ of tumors. In the cases with a change in hormone receptor status there was a higher proportion of tumors with increased Ki-67 index as compared with tumors showing no change in hormone receptor status. Additionally, the 5-year DFS and OS rates were significantly lower in the group with change in hormone receptor status $(43.2 \%$ and $60.4 \%$, respectively) as compared to the group with no change (67.9\% and $81.8 \%$, respectively).[22]

Surg Oncol Clin N Am. Author manuscript; available in PMC 2015 July 01. 
Similarly, loss of HER2 amplification after neoadjuvant chemotherapy with trastuzumab has been associated with decreased 3-year RFS as compared with patients who retain HER2 amplification $(50.0 \%$ vs. $87.5 \%, \mathrm{p}=0.041)$.[23]

\section{Neoadjuvant therapy in patients with HER2-positive tumors}

Trastuzumab, a humanized monoclonal antibody targeted against the extracellular domain of the human epidermal growth factor receptor 2 (HER2), has revolutionized the treatment of women with HER2-positive breast cancer with increased survival rates reported in the metastatic and adjuvant settings when used in combination with chemotherapy. In the neoadjuvant setting, significantly increased rates of PCR have been observed in patients treated with trastuzumab and chemotherapy versus chemotherapy alone. In a small trial conducted by Buzdar and colleagues, the increased pCR when trastuzumab was combined with chemotherapy was so dramatic $(65.2 \%$ vs. $26.3 \%, p=0.016)$ that after review by the data and safety monitoring board, the control arm of this phase III, randomized study was terminated prematurely.[24]

Several groups have investigated the use of anti-HER2 therapies in the neoadjuvant setting (Table 1).[24-28] In the NeOAdjuvant Herceptin (NOAH) trial, women with locally advanced breast cancer were randomized to trastuzumab (loading dose of $8 \mathrm{mg} / \mathrm{m}^{2}$ followed by 10 cycles of $6 \mathrm{mg} / \mathrm{m}^{2}$ ) in addition to neoadjuvant chemotherapy (four cycles of doxorubicin $60 \mathrm{mg} / \mathrm{m}^{2}$ with paclitaxel $175 \mathrm{mg} / \mathrm{m}^{2}$ followed by 3 cycles of cyclophosphamide $600 \mathrm{mg} / \mathrm{m}^{2}$, methotrexate $60 \mathrm{mg} / \mathrm{m}^{2}$ and fluorouracil $600 \mathrm{mg} / \mathrm{m}^{2}$ ) versus neoadjuvant chemotherapy alone. Trastuzumab was given for one year postoperatively.

Patients receiving neoadjuvant trastuzumab experienced an increased pCR in the breast and axillary lymph nodes as compared to those treated with chemotherapy alone (39\% vs. $20 \%$, $\mathrm{p}=0.001)$. The investigators reported an improved 3-year event-free survival (71\% vs. $56 \%$, $\mathrm{p}=0.013$ ) with low cardiac toxicity in those patients receiving concurrent trastuzumab with chemotherapy.[27] The use of neoadjuvant trastuzumab was also associated with a trend toward increased breast conserving surgery $(23 \%$ vs. $13 \%, \mathrm{p}=0.07)$ without an increase in local recurrence, particularly in patients with non-inflammatory breast cancer and hormone receptor-negative disease.[29]

To address the need for concurrent administration of trastuzumab in combination with anthracyclines in order to achieve high pCR rates, the American College of Surgeons Oncology Group (ACOSOG) Z1041 trial compared pCR rates in the breast for 280 women with operable or locally advanced breast cancer randomized to FEC-75 (5-fluorouracil 500 $\mathrm{mg} / \mathrm{m}^{2}$, epirubicin $75 \mathrm{mg} / \mathrm{m}^{2}$ and cyclophosphamide $500 \mathrm{mg} / \mathrm{m}^{2}$ ) followed by paclitaxel (80 $\mathrm{mg} / \mathrm{m}^{2}$ ) with trastuzumab (arm 1) to women treated with paclitaxel with trastuzumab followed by FEC-75 with trastuzumab (arm 2). There was no difference in pCR rates between the two arms $(48.3 \%$ arm 1 vs. $46.7 \%$ arm $2, \mathrm{p}=0.081)$ suggesting that trastuzumab does not need to be delivered concurrently with anthracyclines to achieve high pCR rates. While cardiac toxicities were low in both arms, further follow-up is needed in order to assess the cardiac safety of these regimens.

More recently, other HER2 targeted therapies have also been examined in the neoadjuvant setting with and without chemotherapy. The HER2 positive arm of the GeparQuinto trial 
compared trastuzumab plus chemotherapy with lapatinib plus chemotherapy in the neoadjuvant setting. This trial randomized 620 women with HER2-positive breast cancer and found pCR rates of $30.3 \%$ in the trastuzumab arm and $22.7 \%$ in the lapatinib arm (OR $0.6895 \%$ CI $0.047-0.97, \mathrm{p}=0.04$ ) concluding that trastuzumab was superior to lapatinib as HER2 directed monotherapy in the neoadjuvant setting.[30]

Other trials have investigated dual HER2 targeting in the neoadjuvant setting with lapatinib, pertuzumab and trastuzumab, all of which have differing mechanisms of action (Table 2) and toxicity (Table 3).[31-35] The NeoALTTO trial, a multicenter phase III trial randomized 455 women to receive either lapatinib, trastuzumab or the combination in addition to paclitaxel. The investigators found significantly increased breast pCR rates (51.3\% vs. $29.5 \%$, OR 2.6 97.5\% CI 1.50-4.58, $\mathrm{p}=0.0001)$, breast and axilla pCR rates (OR 2.39 97.5\% CI 1.36-4.26, p = 0.0007) and an increased proportion of patients with pathologically negative axillary lymph nodes after surgery $(73 \%$ vs. $58.6 \%, p=0.0115)$ in the lapatinib plus trastuzumab arm as compared to the trastuzumab only arm. There was no difference between the lapatinib only and trastuzumab only arms. Toxicity was highest in the lapatinib treated groups with diarrhea and hepatic toxicity limiting therapy or prompting dose adjustments.[31] Similarly, the NeoSphere study, a randomized multicenter phase II trial investigating the addition of pertuzumab to trastuzumab with or without docetaxel found a doubling of pCR rates in women treated with dual HER2 therapy in comparison with trastuzumab and chemotherapy alone $(45.8 \%$ vs. $29 \%, \mathrm{p}=0.0141)$. Of note, in the treatment arm where patients received dual HER 2 targeted therapy without any chemotherapy, the pCR rate was $16.8 \%$, suggesting some patients may benefit from HER2directed therapy alone without chemotherapy.[32]

\section{Neoadjuvant therapy in patients with triple receptor negative breast cancer}

Triple receptor negative breast cancer (TNBC) is distinguished by the lack of expression of estrogen, progesterone and HER2 receptors and uniformly carries a poor prognosis.

Following neoadjuvant chemotherapy, investigators have shown increased pCR rates in patients with TNBC as compared with non-TNBC subtypes ( $22 \%$ vs. $11 \%$ OR $=1.5395 \%$ CI $1.03-2.26, \mathrm{p}=0.034)$. In addition, those patients with TNBC achieving a pCR were found to have comparable overall survival rates to non-TNBC patients. Unfortunately, patients with TNBC who have residual disease after neoadjuvant chemotherapy have a particularly poor prognosis.[36, 37]

The lack of specific targets in patients with TNBC has prompted investigators to examine alternative chemotherapeutic regimens in this population. Several studies have shown improved pCR rates with sequential anthracycline-taxane based neoadjuvant chemotherapy in TNBC.[38] Given the similarities between TNBC and BRCA1-associated cancers, use of platinum containing drug regimens has been of particular interest. These agents promote interstrand DNA cross-linking triggering apoptosis. Another area of investigation has been the use of PARP (poly-ADP-ribose polymerase) inhibitors. These agents compromise DNA repair leading to selective cell death and have shown mixed results in TNBC.[39] The use of these agents in combination with chemotherapy is the subject of ongoing clinical trials in patients with TNBC.

Surg Oncol Clin N Am. Author manuscript; available in PMC 2015 July 01. 


\section{Neoadjuvant therapy in patients with hormone receptor-positive breast cancer}

Numerous studies have documented that patients with ER-positive tumors have decreased clinical and pathologic response rates to neoadjuvant chemotherapy compared with other subtypes. Since the major impact on recurrence and survival in patients with hormone receptor positive tumors is attributed to endocrine therapies there has been an interest in using this approach in the neoadjuvant setting. While neoadjuvant endocrine therapies have not been associated with significant pCR rates, a significant clinical impact has been demonstrated in down-staging tumors and increasing rates of breast conservation therapy in this population. Expression of the proliferation marker, Ki-67 after short-term exposure to endocrine therapy has also been shown to be a useful tool for assessing clinical benefit.[40]

Patients best suited for neoadjuvant endocrine therapy are postmenopausal women with large or locally advanced ER-positive tumors, elderly women who are unable to tolerate toxicities associated with chemotherapy or are not surgical candidates secondary to significant co-morbidities. To date, a role for neoadjuvant endocrine therapy has not been established in the treatment of premenopausal women with breast cancer. Early trials with endocrine therapy primarily utilized tamoxifen, a competitive inhibitor of estradiol which binds to the estrogen receptor. Aromatase inhibitors, which inactivate the aromatase enzyme thereby inhibiting the conversion of adrenal androgens to estrogen, have also been investigated in the neoadjuvant setting.

Several trials have compared the efficacy of tamoxifen and aromatase inhibitors in the neoadjuvant setting (Table 4).[41-43] A multicenter study evaluating 324 postmenopausal women with ER and/or PR positive breast cancer randomized participants to tamoxifen 20 $\mathrm{mg}$ daily or letrozole $2.5 \mathrm{mg}$ daily for 4 months followed by surgery. No women were considered breast conservation therapy candidates at baseline. The investigators found a superior overall response (both complete and partial response) in the letrozole group (55\% vs. $36 \%, \mathrm{p}<0.001)$. Also significant was an increased conversion to breast conservation therapy in the letrozole group ( $45 \%$ vs. $35 \%, \mathrm{p}=0.022)$.[41]

Similar findings were noted in the Immediate Preoperative Anastrozole, Tamoxifen or Combined with Tamoxifen (IMPACT) trial, which examined the efficacy of anastrozole versus tamoxifen and a combination of anastrozole and tamoxifen in the neoadjuvant setting. Three hundred thirty postmenopausal women with ER-positive, operable or locally advanced breast cancer were randomized to one of 3 treatment arms (tamoxifen $20 \mathrm{mg}$ daily, anastrozole $1 \mathrm{mg}$ daily or both tamoxifen $20 \mathrm{mg}$ and anastrozole $1 \mathrm{mg}$ daily) and treated for 3 months prior to surgery. There was no difference in overall response rates (by clinical exam and ultrasound) or conversion to breast conservation rates among the groups. However, when tamoxifen was compared with anastrozole there was an improvement in breast conservation from $22 \%$ to $46 \%$, respectively (OR 2.94 CI 1.11 to $7.81, \mathrm{p}=0.03$ ). No difference was observed when comparing tamoxifen with the combination of anastrozole and tamoxifen. The investigators also found an increased response to therapy with increased ER levels $(p=0.02)$.[42] 
These studies demonstrated the superiority of aromatase inhibitors over tamoxifen as primary endocrine therapy for postmenopausal women with estrogen receptor-positive breast cancer in down-staging tumors and allowing for breast conservation.

The ACOSOG Z1031 phase II trial directly compared all three aromatase inhibitors (exemestane, letrozole and anastrozole) in the neoadjuvant setting. This study analyzed 374 postmenopausal women with stage II or III ER-positive breast cancer randomized to receive one of the aromatase inhibitors for 16 weeks. The investigators found similar clinical response rates among the three agents with the highest response observed with letrozole treatment (74.8\% compared with $62.9 \%$ with exemestane and $69.1 \%$ with anastrozole). There were similar rates of BCT in each group. The three treatments were also similar in biologic effect as evidenced by mean percentage decrease in Ki-67 from baseline to that measured after therapy.[44]

There is limited data comparing neoadjuvant endocrine therapy with neoadjuvant chemotherapy. Semiglazov et al., reported results from a randomized phase II trial comparing 239 postmenopausal women with ER-positive stage IIA to stage IIIB breast cancer randomized to endocrine therapy (exemestane $25 \mathrm{mg}$ daily or anastrozole $1 \mathrm{mg}$ daily) or chemotherapy (four cycles of doxorubicin $60 \mathrm{mg} / \mathrm{m}^{2}$ and paclitaxel $200 \mathrm{mg} / \mathrm{m}^{2}$ ) for three months prior to surgery. The investigators found no statistically significant difference in overall objective response between the neoadjuvant chemotherapy and neoadjuvant endocrine groups. The pathologic complete response rate was $6 \%$ and 3\%, respectively. There was also no difference in breast conservation or local recurrence at 36 months. A higher rate of adverse events was noted in the neoadjuvant chemotherapy group. This study suggests that neoadjuvant aromatase inhibitor therapy is similar to neoadjuvant chemotherapy in postmenopausal women with ER-positive tumors in terms of clinical and pathologic response rates and confers less toxicity.[45]

\section{Poor response to neoadjuvant chemotherapy}

Unfortunately, the majority of patients do not achieve pCR with neoadjuvant therapy and studies have consistently demonstrated reduced survival outcomes in those patients with residual disease. Those with disease progression on therapy have an unfavorable prognosis and present a clinical challenge with limited therapeutic options to improve outcome. Early identification of poor response or disease progression is important to spare patients the toxicity of ineffective therapy. Once identified, this typically prompts surgical intervention or a change in chemotherapeutic regimen.

The benefit of switching to a non-cross resistant regimen has not been demonstrated in clinical trials. The GeparTrio trial studied the impact of changing chemotherapeutic regimens in patients who showed non-responsiveness early in the neoadjuvant course. This phase III study enrolled 2,072 women, $67.1 \%$ of which demonstrated response to therapy. The remaining 622 women who did not respond to two initial cycles of TAC (doxorubicin $50 \mathrm{mg} / \mathrm{m}^{2}$, cyclophosphamide $500 \mathrm{mg} / \mathrm{m}^{2}$ and docetaxel at $75 \mathrm{mg} / \mathrm{m}^{2}$ ) were randomized to four additional cycles of TAC or four cycles of NX (vinorelbine $25 \mathrm{mg} / \mathrm{m}^{2}$ and capcecitabine $1000 \mathrm{mg} / \mathrm{m}^{2}$ ). Similar efficacy between the two treatment arms was observed with pCR rates of 5.3\% in the TAC group and 6.0\% in the NX group.[46] These patients, 
classified as non-responders, show decreased responsiveness to neoadjuvant chemotherapy likely secondary to a lack of inherent chemosensitivity conferring a worse outcome. By comparison, responders achieved a pCR rate of $21.0 \%$ after six additional cycles of TAC and $23.5 \%$ after eight additional cycles of TAC.[47]

There are currently efforts underway to determine genetic changes that may identify resistant tumors at diagnosis so that these patients might be targeted for novel agents. In addition, biomarkers are being investigated that can be used to direct therapies in patients with significant residual disease after neoadjuvant therapy.

\section{Monitoring of response to neoadjuvant therapy}

During the neoadjuvant treatment course patients are monitored with physical examination to assess changes in the primary tumor and regional lymph nodes. There are currently no established recommendations for imaging surveillance during therapy.[11] The pretreatment placement of radiopaque clips in the primary tumor for patients planning to pursue breast conservation is important for tumor localization in the setting of a complete radiographic response and has been associated with improved local control.[48] Upon the completion of neoadjuvant therapy, the residual tumor burden is typically estimated by clinical and radiographic methods to assess response.

The combination of mammography and ultrasound has been shown to better correlate with pCR than physical examination alone with a sensitivity and specificity in predicting pCR of $78.6 \%$ and $92.5 \%$, respectively. However, pathologic residual may vary with underlying tumor biology and specifically may be underestimated with invasive lobular histology and overestimated with poorly differentiated tumors.[49]

Contrast-enhanced breast MRI has shown promise in predicting response after neoadjuvant chemotherapy. In the ACRIN 6657/I-SPY trial, investigators were able to demonstrate superiority of MRI as compared to physical examination in predicting response to therapy, with the greatest predictive ability in change in tumor volume occurring early in the treatment course.[50] MRI has also been studied with respect to its predictive power in detecting patients with pCR. Evaluation of radiographic complete response (rCR) by MRI and pCR in the Translational Breast Cancer Research Consortium (TBCRC) Trial 017, found an overall accuracy of $74 \%$ for MRI in predicting pCR in 746 patients after neoadjuvant chemotherapy for breast cancer. Triple negative subtype, HER2-positive tumors and lower $\mathrm{T}$ classification at presentation were found to be independently associated with increased rCR. Similarly, these variables were found to be associated with achieving pCR with the addition of African-American race.[51]

The impact of breast imaging in measuring early response to neoadjuvant therapy and predicting pCR may be a powerful tool informing a change in systemic therapy for poor responders or possibly identifying a subset of patients in association with tumor subtype who will not ultimately require surgical intervention.

Surg Oncol Clin N Am. Author manuscript; available in PMC 2015 July 01. 


\section{Impact of neoadjuvant chemotherapy on local-regional therapies}

\section{Breast conservation therapy (BCT)}

An established benefit of neoadjuvant systemic therapy is a decrease in tumor burden such that patients with large tumors become candidates for breast conservation. This is contingent on the patient's desire for breast conservation, candidacy for adjuvant radiotherapy and consideration of cosmesis. Factors associated with decreased feasibility for BCT include lobular histology, multicentricity and diffuse calcifications on mammography.[52]

The primary concern in breast conservation after neoadjuvant chemotherapy is the risk of local-regional recurrence. The NSABP B-18 trial suggested a slight increase in ipsilateral breast tumor recurrence in the neoadjuvant group however this difference was not statistically significant.[4] Increased local recurrence after neoadjuvant chemotherapy has been described however, primarily in the setting of radiotherapy alone without surgical intervention in patients with complete clinical response.[7] This highlights the distinction that complete clinical response does not confer $\mathrm{pCR}$ and the critical role for operative intervention in achieving local control. When breast conserving surgery is followed with whole breast radiation therapy several studies have shown no significant increase in local recurrence after neoadjuvant chemotherapy. Furthermore, prior treatment with neoadjuvant chemotherapy does not compromise salvage after local-regional recurrence in these patients. [53]

To better identify selection criteria for BCT after neoadjuvant chemotherapy, four statistically significant predictors of ipsilateral breast tumor recurrence (IBTR) and localregional recurrence (LRR) were utilized to develop the MD Anderson prognostic index (MDAPI). These were clinical N2 or N3 disease, residual pathologic tumor size $>2 \mathrm{~cm}$, a multifocal pattern of residual disease and lymphovascular space invasion. Each variable, if present, is assigned a score of 1 and calculated into an overall score. Stratification by low risk (score 0-1), intermediate risk (score 2), and high risk (score 3-4) resulted in a 5-year IBTR-free survival of $97 \%, 88 \%$, and $82 \%$, respectively $(\mathrm{p}=0.0001)$ and 5-year LRR-free survival of $94 \%, 83 \%$ and $58 \%$, respectively $(\mathrm{p}=0.0001) .[54,55]$

Additionally, local-regional recurrence after neoadjuvant chemotherapy is influenced by tumor subtype following BCT or mastectomy. Specifically, the basal subtype (ER/PRnegative/HER2-negative) appears to be associated with increased LRR as compared to luminal A (ER/PR-positive/HER2-negative), luminal B (ER/PR-positive/HER2-positive) and HER2 enriched (ER/PR-negative/HER2-positive) subtypes $(p=0.03)$.[56]

\section{Nodal assessment after chemotherapy}

In addition to the primary tumor, neoadjuvant chemotherapy also has impact on downstaging or eradicating disease in the axillary lymph nodes. Pathologically negative axillary nodes after neoadjuvant chemotherapy remains a strong predictor of prognosis.[5] In this setting, concerns initially arose over the identification and accuracy of sentinel lymph node (SLN) dissection in staging the axilla following neoadjuvant chemotherapy. Reports from many single institution studies have shown the utility and safety of SLN dissection after neoadjuvant chemotherapy in clinically node-negative patients with comparable

Surg Oncol Clin N Am. Author manuscript; available in PMC 2015 July 01. 
identification and false negative rates to those undergoing surgery first. Thus, decreasing the role for completion axillary lymph node dissection and sparing the potential associated morbidity in these patients.[57, 58]

In patients with clinically node-positive disease at presentation, the role of sentinel lymph node dissection is more controversial. These patients present a clinical challenge, as approximately $42 \%$ will achieve a pCR with pathologically negative disease at the time of axillary lymph node dissection.[59] The ACOSOG Z1071 trial specifically addressed this question, evaluating 756 women with clinically positive lymph nodes prior to neoadjuvant chemotherapy. All patients underwent SLN dissection and subsequent axillary lymph node dissection. SLN dissection correctly identified nodal status in $91.2 \%$ of patients with a false negative rate of $12.6 \%$. Although this false negative rate was higher than the preset false negative rate defined by the study, the investigators reported that improved accuracy was obtained with use of both blue dye and radioactive tracer and removal of 2 or more sentinel lymph nodes.[60] Furthermore, when the sentinel lymph node correlated with the radiographically suspicious lymph node evidenced by inclusion of a clip marker placed prior to treatment, the false negative rate was reduced to $7.4 \%$.

\section{Reconstructive surgery}

Although neoadjuvant chemotherapy improves rates of breast conservation, many women still require mastectomy for optimal local-regional control. In this population, postmastectomy breast reconstruction is an important multidisciplinary consideration. This is particularly true given, after neoadjuvant chemotherapy women are less likely to undergo immediate reconstruction.[61] One contributing concern limiting immediate reconstruction is the delay of adjuvant therapy, however a single institution retrospective review from the Institut of Gustave-Roussy found no difference in interval from surgery to adjuvant chemotherapy or radiotherapy based on receipt of neoadjuvant chemotherapy or type of reconstruction (implant vs. autologous tissue flap). This study also found no difference in 5year local relapse free survival with respect to timing of reconstruction. The 5- and 10-year distant disease-free survival rates were improved in the group with delayed reconstruction which is postulated to be secondary to the exclusion of patients with metastatic disease from delayed reconstruction.[62]

An additional concern is the potential for increased postoperative complications with reconstruction following chemotherapy and radiation. A study from the Dana-Farber Cancer Institute found no association between neoadjuvant chemotherapy and increased postoperative complications after immediate or delayed reconstruction.[61] Similarly, an investigation utilizing the American College of Surgeons National Surgical Quality Improvement Program (ACS-NSQIP) database found that neoadjuvant chemotherapy did not increase wound complications following breast conservation or mastectomy. They did observe a non-significant trend towards increased wound complications in patients who underwent neoadjuvant chemotherapy and immediate reconstruction after mastectomy.[63] 


\section{Radiation therapy}

While the role of whole breast radiation therapy in BCT is undisputed, postmastectomy radiation therapy (PMRT) after neoadjuvant chemotherapy is not as well studied. There appears to be a benefit of radiation therapy in patients with stage III disease treated with neoadjuvant chemotherapy followed by mastectomy in improving local-regional control even after achieving pCR. In one study, the 10-year LRR rate was $7.3 \%$ after PMRT compared to $33.3 \%$ in those who did not receive radiation $(\mathrm{p}=0.040)$. Additionally, there was a significant difference in overall survival, distant metastasis-free survival and causespecific survival in this population when treated with PMRT.[64]

The NSABP B-51/RTOG 1304 (NRG 9353) study is a phase III trial planned to investigate the role of radiation therapy after neoadjuvant chemotherapy in women with T1-3 N1 disease and pathologically negative axillary nodal disease after treatment. Patients will be randomized to regional lymph node radiation after whole breast radiation for BCT and radiation to the chest wall and regional lymphatics after mastectomy versus no additional radiation.

\section{Summary}

The use of neoadjuvant chemotherapy has evolved from its role in inoperable and locally advanced breast cancer to the treatment of selected patients with early-stage, operable disease. Survival is comparable to treatment with adjuvant systemic therapy, however the neoadjuvant approach carries the benefit of decreasing tumor burden thus facilitating breastconserving surgery and tumor response to therapy holds individualized prognostic value by tumor subtype. Neoadjuvant endocrine therapy with aromatase inhibitors in postmenopausal women with hormone receptor-positive tumors, has a role in tumor down-staging particularly in patients who are not acceptable candidates for chemotherapy or surgery. Neoadjuvant chemotherapy also decreases the incidence of positive axillary lymph nodes resulting in a reduction in the number of patients requiring axillary lymph node dissection on completion of chemotherapy.

Future directions include further refinement of predictors of response to therapy, development of targeted treatments and optimization of chemotherapeutic regimen by subtype. Clinical trials are now addressing the role of additional treatment in patients with residual disease and high-risk subtypes and determination of patients who may not require surgical management after neoadjuvant chemotherapy.

\section{References}

1. Mamounas EP, Fisher B. Preoperative (neoadjuvant) chemotherapy in patients with breast cancer. Semin Oncol. 2001; 28:389-399. [PubMed: 11498832]

2. Fisher B, Gunduz N, Saffer EA. Influence of the interval between primary tumor removal and chemotherapy on kinetics and growth of metastases. Cancer Res. 1983; 43:1488-1492. [PubMed: 6831397]

3. Ragaz J, Baird R, Rebbeck P, et al. Neoadjuvant (preoperative) chemotherapy for breast cancer. Cancer. 1985; 56:719-724. [PubMed: 4016668]

4. Fisher B, Bryant J, Wolmark N, et al. Effect of preoperative chemotherapy on the outcome of women with operable breast cancer. J Clin Oncol. 1998; 16:2672-2685. [PubMed: 9704717]

Surg Oncol Clin N Am. Author manuscript; available in PMC 2015 July 01. 
5. Rastogi P, Anderson SJ, Bear HD, et al. Preoperative chemotherapy: updates of National Surgical Adjuvant Breast and Bowel Project Protocols B-18 and B-27. J Clin Oncol. 2008; 26:778-785. [PubMed: 18258986]

6. van der Hage JA, van de Velde CJ, Julien JP, et al. Preoperative chemotherapy in primary operable breast cancer: results from the European Organization for Research and Treatment of Cancer trial 10902. J Clin Oncol. 2001; 19:4224-4237. [PubMed: 11709566]

7. Mauri D, Pavlidis N, Ioannidis JP. Neoadjuvant versus adjuvant systemic treatment in breast cancer: a meta-analysis. J Natl Cancer Inst. 2005; 97:188-194. [PubMed: 15687361]

8. Mieog JS, van der Hage JA, van de Velde CJ. Neoadjuvant chemotherapy for operable breast cancer. Br J Surg. 2007; 94:1189-1200. [PubMed: 17701939]

9. Kuerer HM, Newman LA, Smith TL, et al. Clinical course of breast cancer patients with complete pathologic primary tumor and axillary lymph node response to doxorubicin-based neoadjuvant chemotherapy. J Clin Oncol. 1999; 17:460-469. [PubMed: 10080586]

10. Mazouni C, Peintinger F, Wan-Kau S, et al. Residual ductal carcinoma in situ in patients with complete eradication of invasive breast cancer after neoadjuvant chemotherapy does not adversely affect patient outcome. J Clin Oncol. 2007; 25:2650-2655. [PubMed: 17602071]

11. Kaufmann M, von Minckwitz G, Mamounas EP, et al. Recommendations from an international consensus conference on the current status and future of neoadjuvant systemic therapy in primary breast cancer. Ann Surg Oncol. 2012; 19:1508-1516. [PubMed: 22193884]

12. Ju NR, Jeffe DB, Keune J, Aft R. Patient and tumor characteristics associated with breast cancer recurrence after complete pathological response to neoadjuvant chemotherapy. Breast Cancer Res Treat. 2013; 137:195-201. [PubMed: 23149464]

13. Gonzalez-Angulo AM, McGuire SE, Buchholz TA, et al. Factors predictive of distant metastases in patients with breast cancer who have a pathologic complete response after neoadjuvant chemotherapy. J Clin Oncol. 2005; 23:7098-7104. [PubMed: 16192593]

14. Prowell TM, Pazdur R. Pathological complete response and accelerated drug approval in early breast cancer. N Engl J Med. 2012; 366:2438-2441. [PubMed: 22646508]

15. Symmans WF, Peintinger F, Hatzis C, et al. Measurement of residual breast cancer burden to predict survival after neoadjuvant chemotherapy. J Clin Oncol. 2007; 25:4414-4422. [PubMed: 17785706]

16. Jeruss JS, Mittendorf EA, Tucker SL, et al. Combined use of clinical and pathologic staging variables to define outcomes for breast cancer patients treated with neoadjuvant therapy. J Clin Oncol. 2008; 26:246-252. [PubMed: 18056680]

17. Mittendorf EA, Jeruss JS, Tucker SL, et al. Validation of a novel staging system for diseasespecific survival in patients with breast cancer treated with neoadjuvant chemotherapy. J Clin Oncol. 2011; 29:1956-1962. [PubMed: 21482989]

18. Perou CM, Sorlie T, Eisen MB, et al. Molecular portraits of human breast tumours. Nature. 2000; 406:747-752. [PubMed: 10963602]

19. Houssami N, Macaskill P, von Minckwitz G, et al. Meta-analysis of the association of breast cancer subtype and pathologic complete response to neoadjuvant chemotherapy. Eur J Cancer. 2012; 48:3342-3354. [PubMed: 22766518]

20. Guarneri V, Broglio K, Kau SW, et al. Prognostic value of pathologic complete response after primary chemotherapy in relation to hormone receptor status and other factors. J Clin Oncol. 2006; 24:1037-1044. [PubMed: 16505422]

21. von Minckwitz G, Untch M, Blohmer JU, et al. Definition and impact of pathologic complete response on prognosis after neoadjuvant chemotherapy in various intrinsic breast cancer subtypes. J Clin Oncol. 2012; 30:1796-1804. [PubMed: 22508812]

22. Chen S, Chen CM, Yu KD, et al. Prognostic value of a positive-to-negative change in hormone receptor status after neoadjuvant chemotherapy in patients with hormone receptor-positive breast cancer. Ann Surg Oncol. 2012; 19:3002-3011. [PubMed: 22437200]

23. Mittendorf EA, Wu Y, Scaltriti M, et al. Loss of HER2 amplification following trastuzumab-based neoadjuvant systemic therapy and survival outcomes. Clin Cancer Res. 2009; 15:7381-7388.

[PubMed: 19920100]

Surg Oncol Clin N Am. Author manuscript; available in PMC 2015 July 01. 
24. Buzdar AU, Ibrahim NK, Francis D, et al. Significantly higher pathologic complete remission rate after neoadjuvant therapy with trastuzumab, paclitaxel, and epirubicin chemotherapy: results of a randomized trial in human epidermal growth factor receptor 2-positive operable breast cancer. $\mathrm{J}$ Clin Oncol. 2005; 23:3676-3685. [PubMed: 15738535]

25. Buzdar AU, Valero V, Ibrahim NK, et al. Neoadjuvant therapy with paclitaxel followed by 5fluorouracil, epirubicin, and cyclophosphamide chemotherapy and concurrent trastuzumab in human epidermal growth factor receptor 2-positive operable breast cancer: an update of the initial randomized study population and data of additional patients treated with the same regimen. Clin Cancer Res. 2007; 13:228-233. [PubMed: 17200359]

26. Untch M, Rezai M, Loibl S, et al. Neoadjuvant treatment with trastuzumab in HER2-positive breast cancer: results from the GeparQuattro study. J Clin Oncol. 2010; 28:2024-2031. [PubMed: 20308670]

27. Gianni L, Eiermann W, Semiglazov V, et al. Neoadjuvant chemotherapy with trastuzumab followed by adjuvant trastuzumab versus neoadjuvant chemotherapy alone, in patients with HER2positive locally advanced breast cancer (the NOAH trial): a randomised controlled superiority trial with a parallel HER2-negative cohort. Lancet. 2010; 375:377-384. [PubMed: 20113825]

28. Untch M, Fasching PA, Konecny GE, et al. Pathologic complete response after neoadjuvant chemotherapy plus trastuzumab predicts favorable survival in human epidermal growth factor receptor 2-overexpressing breast cancer: results from the TECHNO trial of the AGO and GBG study groups. J Clin Oncol. 2011; 29:3351-3357. [PubMed: 21788566]

29. Semiglazov V, Eiermann W, Zambetti M, et al. Surgery following neoadjuvant therapy in patients with HER2-positive locally advanced or inflammatory breast cancer participating in the NeOAdjuvant Herceptin (NOAH) study. Eur J Surg Oncol. 2011; 37:856-863. [PubMed: 21843921]

30. Untch M, Loibl S, Bischoff J, et al. Lapatinib versus trastuzumab in combination with neoadjuvant anthracycline-taxane-based chemotherapy (GeparQuinto, GBG 44): a randomised phase 3 trial. Lancet Oncol. 2012; 13:135-144. [PubMed: 22257523]

31. Baselga J, Bradbury I, Eidtmann H, et al. Lapatinib with trastuzumab for HER2-positive early breast cancer (NeoALTTO): a randomised, open-label, multicentre, phase 3 trial. Lancet. 2012; 379:633-640. [PubMed: 22257673]

32. Gianni L, Pienkowski T, Im YH, et al. Efficacy and safety of neoadjuvant pertuzumab and trastuzumab in women with locally advanced, inflammatory, or early HER2-positive breast cancer (NeoSphere): a randomised multicentre, open-label, phase 2 trial. Lancet Oncol. 2012; 13:25-32. [PubMed: 22153890]

33. Guarneri V, Frassoldati A, Bottini A, et al. Preoperative chemotherapy plus trastuzumab, lapatinib, or both in human epidermal growth factor receptor 2-positive operable breast cancer: results of the randomized phase II CHER-LOB study. J Clin Oncol. 2012; 30:1989-1995. [PubMed: 22493419]

34. Rimawi MF, Mayer IA, Forero A, et al. Multicenter phase II study of neoadjuvant lapatinib and trastuzumab with hormonal therapy and without chemotherapy in patients with human epidermal growth factor receptor 2-overexpressing breast cancer: TBCRC 006. J Clin Oncol. 2013; 31:17261731. [PubMed: 23569315]

35. Schneeweiss A, Chia S, Hickish T, et al. Pertuzumab plus trastuzumab in combination with standard neoadjuvant anthracycline-containing and anthracycline-free chemotherapy regimens in patients with HER2-positive early breast cancer: a randomized phase II cardiac safety study (TRYPHAENA). Ann Oncol. 2013; 24:2278-2284. [PubMed: 23704196]

36. Liedtke C, Mazouni C, Hess KR, et al. Response to neoadjuvant therapy and long-term survival in patients with triple-negative breast cancer. J Clin Oncol. 2008; 26:1275-1281. [PubMed: 18250347]

37. Carey LA, Dees EC, Sawyer L, et al. The triple negative paradox: primary tumor chemosensitivity of breast cancer subtypes. Clin Cancer Res. 2007; 13:2329-2334. [PubMed: 17438091]

38. Nahleh Z. Neoadjuvant chemotherapy for "triple negative" breast cancer: a review of current practice and future outlook. Med Oncol. 2010; 27:531-539. [PubMed: 19513864]

39. Chang HR, Glaspy J, Allison MA, et al. Differential response of triple-negative breast cancer to a docetaxel and carboplatin-based neoadjuvant treatment. Cancer. 2010; 116:4227-4237. [PubMed: 20549829] 
40. Dowsett M, Smith IE, Ebbs SR, et al. Prognostic value of Ki67 expression after short-term presurgical endocrine therapy for primary breast cancer. J Natl Cancer Inst. 2007; 99:167-170. [PubMed: 17228000]

41. Eiermann W, Paepke S, Appfelstaedt J, et al. Preoperative treatment of postmenopausal breast cancer patients with letrozole: A randomized double-blind multicenter study. Ann Oncol. 2001; 12:1527-1532. [PubMed: 11822750]

42. Smith IE, Dowsett M, Ebbs SR, et al. Neoadjuvant treatment of postmenopausal breast cancer with anastrozole, tamoxifen, or both in combination: the Immediate Preoperative Anastrozole, Tamoxifen, or Combined with Tamoxifen (IMPACT) multicenter double-blind randomized trial. J Clin Oncol. 2005; 23:5108-5116. [PubMed: 15998903]

43. Cataliotti L, Buzdar AU, Noguchi S, et al. Comparison of anastrozole versus tamoxifen as preoperative therapy in postmenopausal women with hormone receptor-positive breast cancer: the Pre-Operative "Arimidex" Compared to Tamoxifen (PROACT) trial. Cancer. 2006; 106:20952103. [PubMed: 16598749]

44. Ellis MJ, Suman VJ, Hoog J, et al. Randomized phase II neoadjuvant comparison between letrozole, anastrozole, and exemestane for postmenopausal women with estrogen receptor-rich stage 2 to 3 breast cancer: clinical and biomarker outcomes and predictive value of the baseline PAM50-based intrinsic subtype--ACOSOG Z1031. J Clin Oncol. 2011; 29:2342-2349. [PubMed: 21555689]

45. Semiglazov VF, Semiglazov VV, Dashyan GA, et al. Phase 2 randomized trial of primary endocrine therapy versus chemotherapy in postmenopausal patients with estrogen receptorpositive breast cancer. Cancer. 2007; 110:244-254. [PubMed: 17538978]

46. von Minckwitz G, Kummel S, Vogel P, et al. Neoadjuvant vinorelbine-capecitabine versus docetaxel-doxorubicin-cyclophosphamide in early nonresponsive breast cancer: phase III randomized GeparTrio trial. J Natl Cancer Inst. 2008; 100:542-551. [PubMed: 18398097]

47. von Minckwitz G, Kummel S, Vogel P, et al. Intensified neoadjuvant chemotherapy in earlyresponding breast cancer: phase III randomized GeparTrio study. J Natl Cancer Inst. 2008; 100:552-562. [PubMed: 18398094]

48. Oh JL, Nguyen G, Whitman GJ, et al. Placement of radiopaque clips for tumor localization in patients undergoing neoadjuvant chemotherapy and breast conservation therapy. Cancer. 2007; 110:2420-2427. [PubMed: 17941034]

49. Peintinger F, Kuerer HM, Anderson K, et al. Accuracy of the combination of mammography and sonography in predicting tumor response in breast cancer patients after neoadjuvant chemotherapy. Ann Surg Oncol. 2006; 13:1443-1449. [PubMed: 17028770]

50. Hylton NM, Blume JD, Bernreuter WK, et al. Locally advanced breast cancer: MR imaging for prediction of response to neoadjuvant chemotherapy--results from ACRIN 6657/I-SPY TRIAL. Radiology. 2012; 263:663-672. [PubMed: 22623692]

51. De Los Santos JF, Cantor A, Amos KD, et al. Magnetic resonance imaging as a predictor of pathologic response in patients treated with neoadjuvant systemic treatment for operable breast cancer. Translational Breast Cancer Research Consortium trial 017. Cancer. 2013; 119:1776-1783. [PubMed: 23436342]

52. Newman LA, Buzdar AU, Singletary SE, et al. A prospective trial of preoperative chemotherapy in resectable breast cancer: predictors of breast-conservation therapy feasibility. Ann Surg Oncol. 2002; 9:228-234. [PubMed: 11923128]

53. Weksberg DC, Allen PK, Hoffman KE, et al. Outcomes and predictive factors for salvage therapy after local-regional recurrence following neoadjuvant chemotherapy and breast conserving therapy. Ann Surg Oncol. 2013; 20:3430-3437. [PubMed: 23720073]

54. Chen AM, Meric-Bernstam F, Hunt KK, et al. Breast conservation after neoadjuvant chemotherapy. Cancer. 2005; 103:689-695. [PubMed: 15641036]

55. Akay CL, Meric-Bernstam F, Hunt KK, et al. Evaluation of the MD Anderson Prognostic Index for local-regional recurrence after breast conserving therapy in patients receiving neoadjuvant chemotherapy. Ann Surg Oncol. 2012; 19:901-907. [PubMed: 21861223] 
56. Meyers MO, Klauber-Demore N, Ollila DW, et al. Impact of breast cancer molecular subtypes on locoregional recurrence in patients treated with neoadjuvant chemotherapy for locally advanced breast cancer. Ann Surg Oncol. 2011; 18:2851-2857. [PubMed: 21442348]

57. Hunt KK, Yi M, Mittendorf EA, et al. Sentinel lymph node surgery after neoadjuvant chemotherapy is accurate and reduces the need for axillary dissection in breast cancer patients. Ann Surg. 2009; 250:558-566. [PubMed: 19730235]

58. Tan VK, Goh BK, Fook-Chong S, et al. The feasibility and accuracy of sentinel lymph node biopsy in clinically node-negative patients after neoadjuvant chemotherapy for breast cancer--a systematic review and meta-analysis. J Surg Oncol. 2011; 104:97-103. [PubMed: 21456092]

59. Alvarado R, Yi M, Le-Petross H, et al. The role for sentinel lymph node dissection after neoadjuvant chemotherapy in patients who present with node-positive breast cancer. Ann Surg Oncol. 2012; 19:3177-3184. [PubMed: 22772869]

60. Boughey JC, Suman VJ, Mittendorf EA, et al. Sentinel lymph node surgery after neoadjuvant chemotherapy in patients with node-positive breast cancer: the ACOSOG Z1071 (Alliance) clinical trial. JAMA. 2013; 310:1455-1461. [PubMed: 24101169]

61. Hu YY, Weeks CM, In H, et al. Impact of neoadjuvant chemotherapy on breast reconstruction. Cancer. 2011; 117:2833-2841. [PubMed: 21264833]

62. Gouy S, Rouzier R, Missana MC, et al. Immediate reconstruction after neoadjuvant chemotherapy: effect on adjuvant treatment starting and survival. Ann Surg Oncol. 2005; 12:161-166. [PubMed: 15827797]

63. Decker MR, Greenblatt DY, Havlena J, et al. Impact of neoadjuvant chemotherapy on wound complications after breast surgery. Surgery. 2012; 152:382-388. [PubMed: 22739071]

64. McGuire SE, Gonzalez-Angulo AM, Huang EH, et al. Postmastectomy radiation improves the outcome of patients with locally advanced breast cancer who achieve a pathologic complete response to neoadjuvant chemotherapy. Int J Radiat Oncol Biol Phys. 2007; 68:1004-1009. [PubMed: 17418973] 


\section{Key points}

- Neoadjuvant chemotherapy in the treatment of breast cancer offers comparable survival benefits to adjuvant chemotherapy.

- Response to neoadjuvant systemic therapy and molecular subtype hold important implications for prognosis and individualized patient outcomes.

- The addition of trastuzumab to neoadjuvant chemotherapy improves pathologic complete response in patients with HER2-positive breast cancer.

- Neoadjuvant endocrine therapy with aromatase inhibitors in postmenopausal women with hormone receptor-positive tumors can significantly decrease tumor burden thereby facilitating breast conserving surgery.

- Neoadjuvant chemotherapy safely allows for a more limited operative approach with breast conserving surgery and sentinel lymph node dissection without an increase in local-regional recurrence rates in selected patients. 


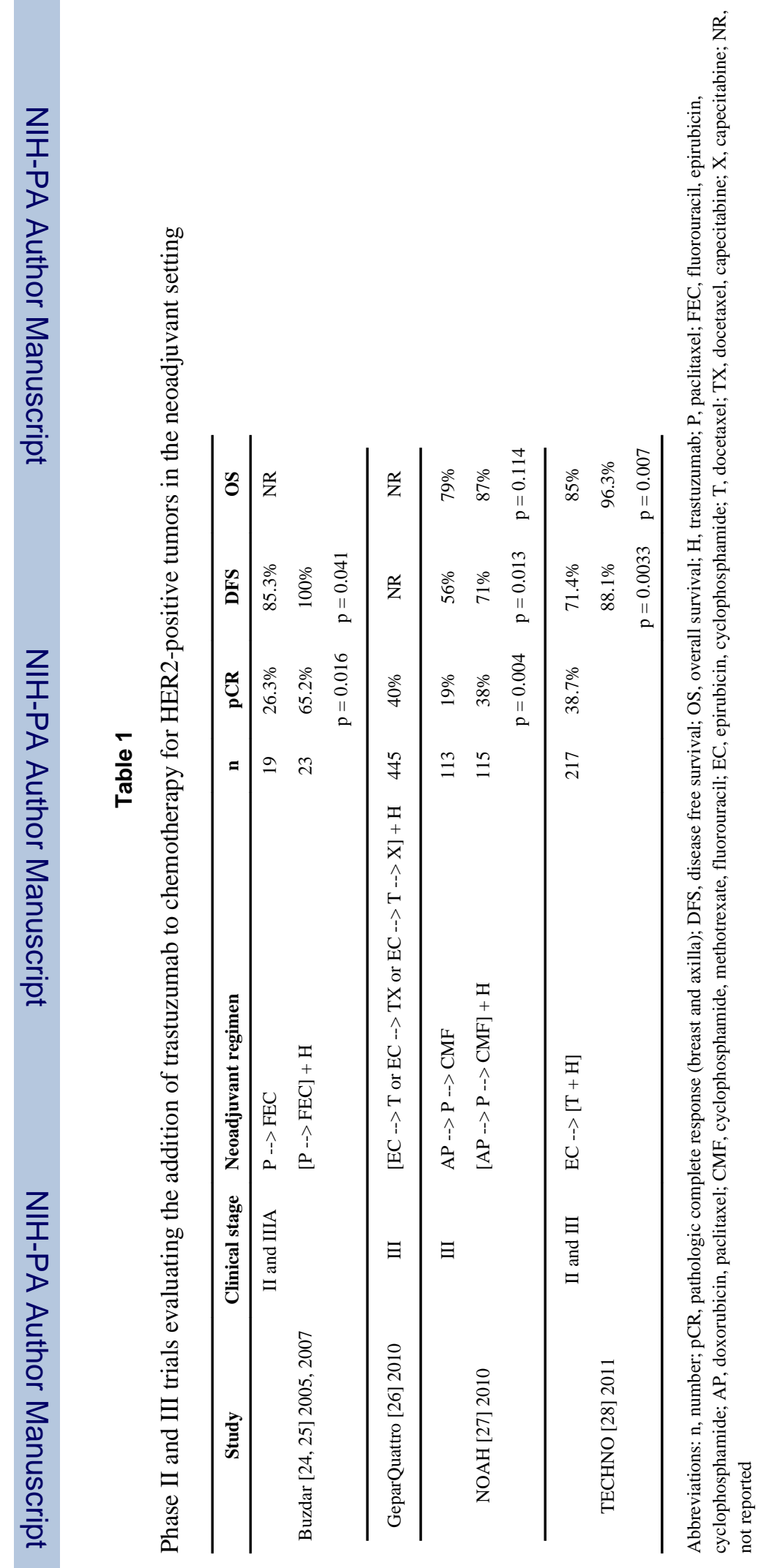




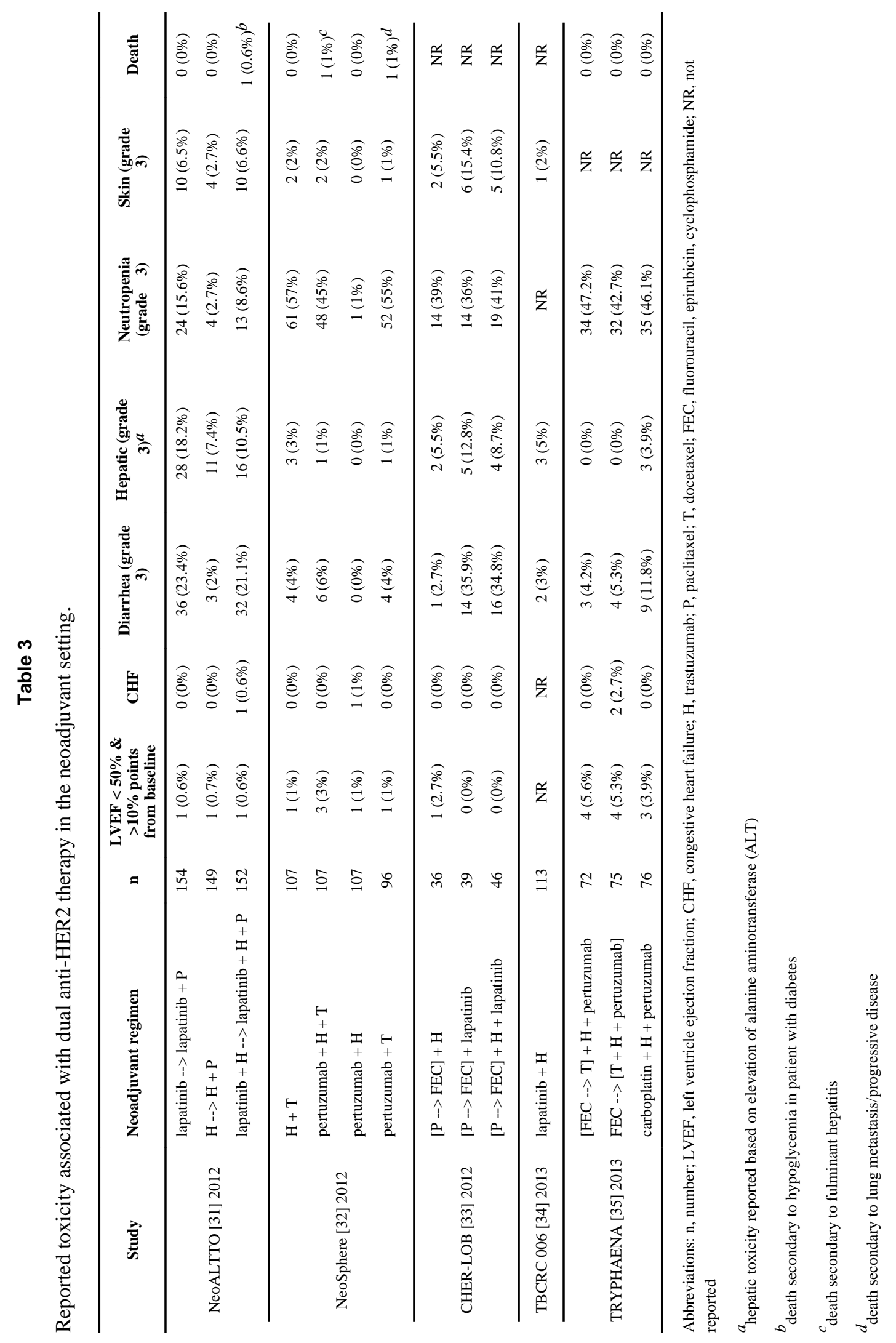




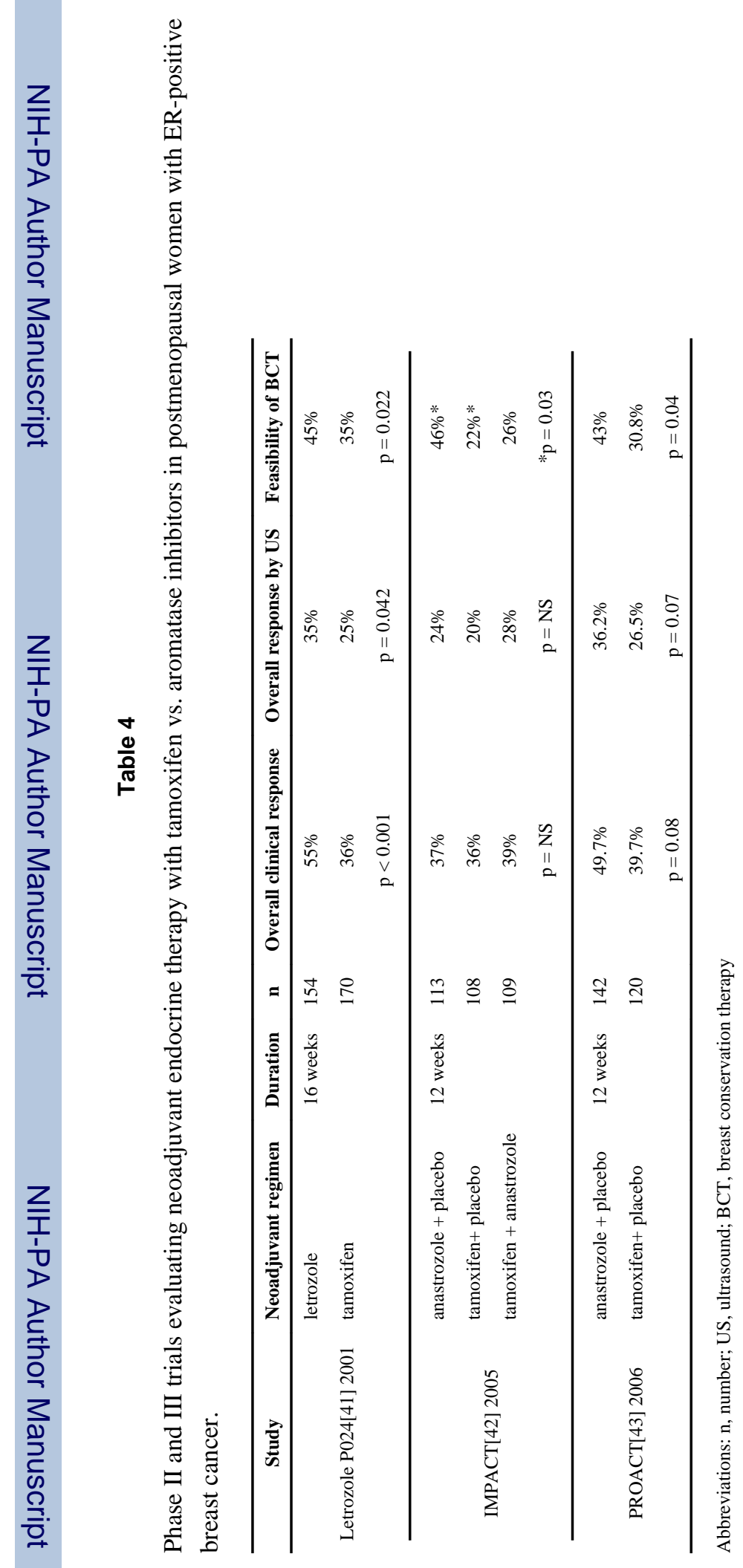

